\title{
Le statut de l'enfant procréé artificiellement : disparités internationales
}

\begin{abstract}
A la suite des colloques internationaux organisés en 1985 à Paris et Lausanne, les auteurs commentent d'un point de vue éthique et psychologique les disparités internationales des lois et réglementations sur la procréation artificielle.
\end{abstract}

\section{Geneviève Delaisi de Parseval \\ Psychanalyste, chercheur en bio- éthique.}

Anne Fagot-Largeault

Maître de conférences de philosophie à l'université Paris XII, médecin psychiatre attaché à l'hôpital Henri-Mondor à Créteil.

\section{ADRESSES}

G. Delaisi de Parseval : I 18 , rue de Vaugirard, 75006 Paris.

A. Fagot-Largeault : I15-117, rue Saint-Antoine, 75004 Paris. e droit de ne pas avoir les enfants qu'on ne veut pas est aujourd'hui effectif dans les pays qui of frent à leurs citoyen(ne)s un large accès à la contraception et à l'interruption de grossesse; il y naît de moins en moins d'enfants nonvoulus (donc, d'enfants disponibles pour l'adoption). Le droit d'avoir les enfants qu'on désire est défendu avec moins d'unanimité que le précédent. "Avoir un enfant n'est pas un droit imprescriptible de l'être humain, ... l'insémination ne doit être autorisée qu'à la condition d'offrir à l'enfant à naître la possibilité d'une croissance favorable ", dit-on en Suède [I], tandis que dans l'état canadien du Saskatchewan, refuser l'insémination d'une femme célibataire passe pour une mesure discriminatoire, et que la Commission de réf orme des lois de l'Ontario s'est demandé si des citoyens canadiens à qui l'on dénierait l'accès à une technique artificielle de procréation n'auraient pas un recours auprès des
Nations Unies [2]. Dans les pays d'Europe de l'est, le droit d'avoir les enfants qu'on veut, quand on le veut (sauf si cela met la santé en danger), apparaît comme un droit constitutionnel, il implique la gratuité des traitements de la stérilité [3]. Savoir si la Convention européenne des droits de l'homme implique, ou non, un "droit à l'enfant ", est objet de controverse [4]. D'aucuns, en France, jugent "que l'on fait plus de cas de l'enfant désiré que de l'enfant né " [5]. Ce débat est très vif depuis les prises de position de R. Badinter lorsqu'il était Garde des Sceaux [6].

Filiation génétique, filiation volontaire et filiation légale.

Cette incertitude se reflète dans le statut de l'enfant " artificiel ". L'enfant "naturel " souffrit naguère de situations juridiques privilégiant le lien social de filiation. On considéra comme un progrès l'évolution du droit qui, en ajoutant au critère social le critère génétique pour la filiation paternelle, rendit aux 
"bâtards " (enfants rarement désirés) le droit de revendiquer l'héritage de leur père (loi de 1972, en France). Et voici que, paradoxalement, l'enfant né des techniques artificielles de reproduction est, dans bien des cas, un bâtard à qui l'on dénie la "vérité " de sa filiation biologique, au nom d'une volonté de procréer. Qui est l'enfant de qui ? De larges disparités internationales mettent en évidence les hésitations contemporaines. On parlera ici surtout de l'insémination artificielle avec sperme de donneur (IAD), pratique la plus ancienne, la mieux connue, et sur laquelle beaucoup de pays ont déjà pris des mesures législatives ou réglementaires.

Depuis que l'IAD se pratique dans le secret des cabinets médicaux (c'est-à-dire depuis 1920, environ), l'effort des parties a été d'effacer toute trace du lien biologique entre l'enfant né de l'IAD et le donneur de sperme : l'enfant était enregistré à l'état civil comme né du couple dont la femme avait été inséminée, il ignorait souvent lui-même sa filiation "artificielle ", le dossier du donneur anonyme était inaccessible ou inexistant. Juger que ce qui fait la paternité, c'est la volonté d'avoir un enfant, de l'élever, de lui transmettre son patrimoine, c'est se ranger à un critère culturel de la filiation; les anthropologues font observer que ce critère culturel prime dans beaucoup de civilisations, où c'est le système de parenté qui désigne les vrais géniteurs. Il se trouve que dans la nôtre, le système traditionnel de parenté s'est effacé derrière une réalité génétique établie scientifiquement. Toutefois, certains pays ont intentionnellement conservé le critère social pour ce cas, en prévoyant qu'un enfant né d'IAD sera au regard de la loi, non pas l'enfant du donneur, mais celui du conjoint de la femme inséminée, cela de façon irréversible. Le Code civil de la Louisiane (1980) stipule : "le mari ne peut désavouer sa paternité à l'égard d'un enfant né d'une insémination de sa femme à laquelle il a consenti " [3]. Et le Code civil du Québec (I980) : "nul ne peut contester la filiation d'une personne pour le motif qu'elle a été conçue par insémination artificielle " et "le recours en désaveu ou en contesta- tion de paternité n'est pas recevable si l'enfant a été conçu par insémination artificielle, soit des œuvres du mari, soit des œuvres d'un tiers, avec le consentement des époux " [3].

Les difficultés immanentes à ce système tournent autour du consentement du père social, mari ou concubin stable de la femme inséminée. L'IAD est cherchée, en règle générale, comme remède à sa stérilité, ou pour prévenir la transmission par lui-même à l'enfant d'une maladie génétique. Faut-il exiger de lui un consentement écrit et signé, comme on le fait par exemple dans l'état canadien du Yukon, et dans certains états américains? Faut-il le présumer consentant, au risque de le voir un jour apporter la preuve qu'il n'avait pas réellement consenti? Le problème est bien posé dans le rapport sur la reproduction artificielle présenté par la Commission de réforme des lois de l'état canadien de l'Ontario [2] : si la possibilité que le donneur soit investi de la paternité est à tout jamais barrée, et que le mari présumé consentant est autorisé à apporter la preuve de son défaut de consentement, l'inconvénient inévitable (et accepté par la commission ontarienne) est que certains enfants nés d'IAD se retrouvent sans père. La même conséquence existe, naturellement, là où l'insémination est accessible aux femmes célibataires. Ceux que n'effraie pas cette conséquence font observer que, dans beaucoup de pays, l'adoption est permise aux célibataires. Quant au donneur, là où le critère volontariste de la filiation domine (Québec, France, état australien du New South Wales, etc.), sa réalité même est oblitérée.

Le droit de l'enfant à connaître sa filiation génétique.

Tout à l'opposé, en .Allemagne Fédérale, le critère biologique est, en cas de contestation, décisif. Il n'existe pas de législation propre à l'IAD, mais une jurisprudence redoutable pour les donneurs de sperme. Une décision du tribunal fédéral $(\mathrm{BGH})$, en 1983 , implique qu'en cas de désaveu de l'enfant IAD par son père "apparent ", la paternité revient automatiquement au donneur. Le médecin inséminateur est tenu d'informer le donneur que le don de sperme, n'étant pas considéré comme un acte médical, n'est pas couvert par le secret professionnel. L'IAD est assimilée à l'adultère. Le conjoint stérile, même s'il a consenti à l'insémination de sa compagne, peut contester sa paternité (et s'il meurt, ses ascendants peuvent le faire en son nom) dans un certain délai après la naissance de l'enfant. L'enfant lui-même peut contester sa filiation (dans un délai de deux ans à partir du moment où l'IAD lui est connue) en cas de divorce de ses parents, ou de faute reprochée à son père social. Quand la procédure judiciaire aboutit, cet enfant de légitime devient illégitime, et ce rétroactivement depuis sa naissance. Si la paternité du donneur est alors prouvée, la filiation naturelle est rétablie, car " un enfant ne peut rester sans père". Le médecin est tenu de communiquer les informations pertinentes touchant le donneur, et celui-ci "ne peut se soustraire aux conséquences de son don " [7].

Il est compréhensible qu'en RFA, depuis 1983 , les donneurs se soient faits rares, et les hôpitaux publics ne pratiquent plus d'inséminations artificielles. Mais les gynécologues privés la pratiquent. En I 985 , on estimait à plus de 20000 personnes la population née d'IAD en République Fédérale [7]. Le nombre des contestations venues devant la justice est très faible, ce qui suggère que, même dans ces conditions précaires, la volonté des parties l'emporte sur la filiation "automatique et obligatoire " prévue par la loi. En Suisse, où environ une naissance sur cent serait actuellement une naissance IAD, la jurisprudence est inexistante. Pourtant, la Suisse penche pour une attitude proche de celle de la RFA. La réforme du droit de la filiation (1976) y a prévu qu'un mari ne peut désavouer l'enfant IAD s'il avait consenti à l'insémination de sa femme. Mais l'enfant, jusqu'à 2I ans, avec l'aide d'un curateur, y peut désavouer son père social, si ses parents se sont séparés. Au cas où cette action aboutit, l'enfant peut, soit être adopté par le nouveau conjoint de sa mère, soit (peut-être) chercher à établir une filiation avec 
le donneur. Le cas ne s'est pas présenté, mais un juriste suisse éminent a récemment écrit que dans cette hypothèse, le médecin serait alors tenu de révéler l'identité du donneur: "la simple promesse d'anonymat doit céder devant le droit de l'enfant à faire établir un lien de filiation" [8].

\section{Les pères des enfants nés d'insé-} mination artificielle.

L'anonymat du donneur joue donc un rôle crucial là où le critère biologique de la filiation pèse fort. En France, où la population née d'IAD était estimée à 12000 personnes en I985 [9], la loi de I 972 (qui ne mentionne pas l'IAD) a largement ouvert le droit de faire prévaloir la vérité génétique en cas de contestation de paternité. Le tribunal de Nice, en 1976, jugea qu'un mari pouvait désavouer l'enfant né de sa femme après IAD (en apportant la preuve qu'il n'était pas le père génétique), bien qu'il eût préalablement consenti à cette IAD. Cela rejoint sans doute une tendance française à faire de l'insémination un droit de la femme, le consentement du conjoint étant alors secondaire (même si les CECOS - Centres d'étude et de conservation du sperme - se donnent pour règle de réserver l'IAD aux seuls couples). Si, en cas de désaveu par le mari, l'enfant reste sans père, ce n'est aucunement par une impossibilité juridique; c'est parce que la procréation artificielle est médicalisée, et que le corps médical respecte strictement le secret professionnel. Révéler le fait de l'IAD, ou l'identité du donneur, serait pour le médecin un manquement à la déontologie; aucun tribunal ne saurait le contraindre à une telle révélation, parce que le droit de connaître ses origines n'est pas, en France, un droit reconnu. Cependant, le maintien de l'anonymat des donneurs est actuellement en discussion [5].

Sur l'acceptabilité d'inséminer des femmes célibataires ou lesbiennes, chaque médecin prend sa décision en conscience; l'Ordre des Médecins tolère une grande diversité d'attitudes, insistant sur le fait qu'en contrepartie de cette liberté, chaque médecin en ces matières informer ses "patients" de son code d'éthique personnel. Il faut noter, qu'en France, le fait que l'IAD entraîne une déclaration mensongère à l'état civil (s'il est "reconnu" par le concubin de la femme) ne soulève pas d'objection; en Grande-Bretagne, par contre, l'état civil étant en principe "véridique", le rapport Warnock proposait qu'on indique sur le registre que le conjoint est le père de l'enfant grâce à un don (by dona(ion) [4].

Les droits du donneur de gamètes...

Dans l'état australien de Victoria, une loi détaillée (1984) couvrant l'insémination artificielle et la fécondation in vitro (FIV) prévoit qu'en cas de don de gamète, aucun lien de filiation ne peut être établi entre le donneur (ou la donneuse) et l'enfant né grâce au don. Le lien de filiation est automatiquement établi avec le couple receveur, le consentement du conjoint stérile est présumé, ce consentement est irréversible; si le conjoint prouve qu'il n'était pas consentant et qu'il est sans lien génétique avec l'enfant, l'enfant est sans père.

Le lien volontaire l'emporte donc sur le lien biologique. Mais ce dernier n'est pas entièrement gommé. D'abord, rien n'interdit au couple de choisir un donneur connu (frère ou cousin du mari pour l'IAD, par exemple), à condition que celui-ci satisfasse aux critères de sélection habituels (examen du sang et du sperme, caryotype, absence de maladie transmissible). En cas de donneur anonyme, le médecin doit, avant d'engager la procédure médicale, fournir par écrit à la femme receveuse des informations sur le donneur, à l'exception de toute information identifiante. Réciproquement, l'insémination n'est possible qu'avec le consentement écrit du donneur (et de son épouse, s'il en a une); le donneur a le droit de solliciter des informations (non-identifiantes) sur la famille receveuse, sur le devenir des grossesses résultant de l'usage de ses gamètes, sur les enfants issus de ces grossesses. Le dévoilement des identités est même acceptable à tout moment, si les deux parties y ont consenti par écrit.
Il est prévu dans le texte de loi que les intervenants soient guidés par des conseillers qualifiés, et que les méthodes impliquant une FIV soient réservées aux hôpitaux dûment autorisés. Les médecins ou institutions pratiquant la fécondation artificielle sont tenus de conserver des dossiers complets sur les donneurs, les receveurs, les procédures, les résultats; ils ne peuvent refuser de communiquer aux parties concernées les renseignements demandés, dans les limites de confidentialité fixées par la loi. La doctrine du Victoria a été résumée par le Comité qui examina là-bas les problèmes posés par la FIV (I983) : "le législateur a vite fait d'effacer le lien légal entre parent génétique et enfant, mais il ne peut ignorer le droit moral de l'enfant à connaître son origine génétique " [2].

La loi suédoise entrée en vigueur en mars 1985 accorde à l'enfant IAD le droit de connaître son géniteur, tout en statuant que le conjoint de la femme inséminée doit donner son consentement écrit à la procédure, et devient par là irrévocablement le père légal de l'enfant. L'hôpital à qui le couple demandeur s'est adressé (seuls les hôpitaux publics sont habilités en Suède à pratiquer la fécondation artificielle) a la responsabilité de sélectionner le donneur, et de conserver son dossier pendant au moins 70 ans. Lorsqu'il a "atteint une maturité suffisante" l'enfant peut, sur sa demande, et avec l'assistance des services sociaux, obtenir communication des informations contenues dans le dossier (identitité comprise). Le dossier est également accessible, sur requête d'un tribunal, en cas de contestation de paternité, mais en dehors de cette hypothèse le droit d'accès est réservé au seul enfant. Celui-ci a donc la possibilité, comme les enfants adoptifs, de rechercher son père biologique. Cela ne crée entre eux aucun lien formel, puisque la loi exclut que le donneur ait jamais à l'égard de cet enfant les obligations d'un père. C'est néanmoins une éventualité dont le donneur est informé, et qu'il doit pouvoir psychologiquement assumer. Il semble d'ailleurs que, depuis la mise en application de la loi, le profil des donneurs ait changé : le donneur- 
type n'est plus un étudiant, mais un père de famille qui fait ce geste avec l'assentiment explicite de sa femme (G. Ewerlöf, communication personnelle).

C'est sur le modèle de l'adoption que les juristes suédois ont traité la filiation artificielle. G. Ewerlöf, secrétaire de la Commission qui prépara la loi de i 985 , explique que l'ancienne attitude, qui consistait en Suède comme ailleurs à tout faire pour éviter que l'enfant IAD apprenne comment il avait été conçu, n'est plus acceptable au regard de ce qu'on sait aujourd'hui de la psychologie des enfants adoptés [io]. Non seulement l'enfant a droit à un père, parce que la présence d'une figure paternelle est aussi nécessaire à son développement que celle d'une figure maternelle (la loi de 85 exclut l'insémination de femmes seules ou lesbiennes), mais il a droit à la franchise sur ses origines. Certes, les parents ne sont pas forcés de révéler à l'enfant qu'il est ne d'une IAD, et le fait de l'IAD n'est pas inscrit à l'état civil. Mais les médecins sont invités, lors de la consultation préalable à la procédure médicale, à apprécier si le couple demandeur est en mesure d'offrir à l'enfant des conditions de croissance favorables, en insistant sur l'importance pour l'équilibre de l'enfant de ne pas lui dissimuler sa conception artificielle. On vise par là, au nom des intérêts de l'enfant, à dissiper l'opprobre qui s'attache à la stérilité masculine (et qui la mure dans le secret). On en accepte la conséquence, qui est de mettre au grand jour la réalité d'une double paternité sociale et biologique, la loi intervenant pour réserver sans ambiguité à la première les droits et devoirs de la fonction (obligation alimentaire, éducation, etc.).

Mère génétique, mère porteuse et mère sociale...

Notons que le critère biologique n'est pas le même pour l'homme et la femme. La contestation de maternité pour absence de lien génétique n'existe nulle part, et aucune donneuse d'ovule ne risque, à ce jour, d'être déclarée mère légale de l'enfant issu de son don. Partout la femme qui accouche de l'enfant est sa mère au regard de la loi, même si dans quelques pays (comme en France) la possibilité d'accoucher anonymement fait qu'il y a des enfants sans mère. Et la femme qui déclare sien à l'état civil un enfant dont une autre a accouché se rend coupable du crime de supposition d'enfant, même si cet enfant est génétiquement d'elle. A notre connaissance, seule la Commission de réforme des lois de l'Ontario a jusqu'ici envisagé que l'enfant né d'une mère de substitution puisse, en toute sécurité juridique, être enregistré à l'état civil comme enfant d'une femme qui ne l'aurait pas porté.

Si l'on suivait la voie de la franchise ouverte par la Suède, on serait conduit à laisser apparaître une maternité triple (mère génétique, mère porteuse, mère sociale), voire quadruple (mère nourrice). Les juristes tiendraient sans doute à ce que la "vraie" mère soit désignée de façon non ambiguë, mais les autres ne seraient pas cachées [II].

La protection des partenaires de la procréation artificielle.

Quel est le prix à payer pour prendre conscience des liens humains découlant des nouvelles techniques de procréation? Quelles personnes, quelles valeurs sont protégées par les systèmes existants? Qu'en est-il des intérêts de l'enfant, que tout le monde invoque, alors que les études objectives sur le devenir des personnes nées d'IAD sont presque inexistantes?

G. Annas [12] déplorait que la pratique de l'IAD aux États-Unis dans les années 1970 protégeât mieux les intérêts des donneurs et des médecins que ceux de l'enfant à naître et de ses parents. C'est un fait que les partenaires en présence dans la procréation artificielle ne sont pas partout traités de façon égale. Nous

\begin{tabular}{|c|c|c|c|c|c|c|}
\hline \multicolumn{7}{|c|}{$\begin{array}{c}\text { Tableau I } \\
\text { IAD, PROTECTION DES INTERÊTS DES DIFFERENTS PARTENAIRES }\end{array}$} \\
\hline \multirow[b]{2}{*}{ France } & \multicolumn{2}{|c|}{$\begin{array}{l}\text { Donneur } \\
\text { et son épouse }\end{array}$} & \multirow{2}{*}{$\begin{array}{c}\text { Médecin } \\
+ \\
+\end{array}$} & \multicolumn{2}{|c|}{ Parents } & \multirow{2}{*}{$\begin{array}{c}\text { Enfant } \\
- \\
-\end{array}$} \\
\hline & & $\begin{array}{l}\text { épouse } \\
+\end{array}$ & & $\begin{array}{c}\text { père } \\
-\end{array}$ & $\begin{array}{l}\text { mère } \\
+(-)\end{array}$ & \\
\hline RFA & - & - & - & - & - & $+(-)$ \\
\hline Suède & $+(-)$ & $+(-)$ & $+(-)$ & $+(-$ & $+(-)$ & + \\
\hline $\begin{array}{l}\text { Etat de } \\
\text { Victoria } \\
\text { (Australie) }\end{array}$ & + & + & + & + & + & + \\
\hline
\end{tabular}

$+=$ bien protégé: $-=$ peu protégé: $+(-)=$ plus ou moins bien protégé.

Le système adopté par l'état de Victoria (4* ligne) protège de façon efficace les intérêts de tous les protagonistes (le donneur, son épouse, les médecins, les parents, l'enfant). A l'inverse, actuellement en RFA les partenaires sont tous, à l'exception de l'enfant, dans une situation très vulnérable (2 ${ }^{\bullet}$ ligne). La France et la Suède sont dans une position intermédiaire. Les droits de l'enfant sont mieux respectés en Suède qu'en France (4ं colonne). mais les intérêts des donneurs et de leurs conjoints sont mieux protégés en France qu'en Suède (1ro colonne). Les médecins sont efficacement protégés partout, sauf en RFA où les responsabilités qui leur incombent sont très lourdes $\left(2^{\circ}\right.$ colonne $)$. Les parents, enfin, sont traités avec égards par le système adopté dans l'état de Victoria, et mal défendus en RFA. La France et la Suède représentent ici encore des cas intermédiaires. II faut noter cependant qu'en France le père (social) a une position particulièrement fragile ( $3^{e}$ colonne). 


\section{REFERENCES}

I. Ewerlöf G. L'insémination artificielle, législation et débat, traduit du suédois par Lydie Rousscau. Actualités suédoises $1985 ; \mathrm{n}^{\circ} 329$, Paris : Institut Suédois. La procréation artificielle et le droit, Suède; table ronde internationale, 7,8 , 9 novembre 1985 , Institut de Recherches Juridiques Comparatives (IRJC) et Centre National de la Recherche Scientifique (CNRS), Paris, 1985.

2. Ontario Law Reform Commission. Report on Human Artificial Reproduction and related matters. Toronto: Ministry of the Attorney General, 1985 (2 vol.).

3. Institut Suisse de Droit Comparé. Procréation artificielle, génétique et droit. Legislation relating to human artificial procreation (a comprehensive collection of laws and regulations dealing specifically with the subject of the colloquium), compiled by Jan Stepan. Colloquium Lausanne, 29-30 novembre 1985 (polycopié)

4. Warnock M. Report of the Committee of Inquiry into Human Fertilisation and Embryology. London: Her Majesty's Stationery Office, 1984.

5. Le droit, la médecine et la vie (dossier). Le Débat 1985; 36: 15-39.

6. Coester-Waltjen D. La procréation artificielle et le droit, République Fédérale d'Allemagne, traduit de l'allemand par M.-J. Foy-Soubrenie. Table ronde internationale, $7,8,9$ novembre 1985 . Paris: IRJC et CNRS, 1985 (polycopié).

7. Guillod O. La procréation artificielle et le droit, Suisse. Table ronde internationale, 7,8 , 9 novembre 1985. Paris: IRJC et CNRS, 1985 (polycopié).

8. Foyer J. Foy M-J, Khaiat L. La procréation artificielle et le droit, France. Table ronde internationale, 7, 8, 9 novembre 1985. Paris: IR JC et CNRS, 1985 (polycopié).

9. Alnot M-O, Labrusse-Riou C, MandelbaumBleitbreu J, Perol Y, Rosencveig J-P. Les procréations artificielles. Rapport préliminaire à Monsieur le Premier Ministre. Paris: La Documentation Française, 1986.

10. Verdier $\mathbf{P}$. Le secret de l'origine des pupilles de l'État. In: Verdier, Soulé, eds. Le secret sur les origines, problèmes psychologiques, légaux, administratifs. Paris: ESF, 1986, chap. I.

I I. Huet-Weiller D. Les nouveaux modes de procréation, Conférence prononcée à l'Université de Fribourg le 3 juin 1986 , texte à paraitre dans la Revue de Métaphysique et de Morale, 1987.

12. Annas GJ. Artificial insemination: beyond the best interests of the donor. Hastings Center Report 1979; 9,4: 14-5

13. Delaisi de Parseval G. Entendre la stérilité. Projet 1985; 195: 22-32.

14. Labrusse-Riou C. La procréation artificielle : questions de droit? Projet 1985; 195: 1 1 5-28.

15. Alnot M O, Parseval G de, Granet Ph, Da Lage C. Sperme, don, contre-don. Conc Med 1982; 104-18: 2915-8. avons tenté de schématiser dans le Tableau I, p. 485, la façon dont sont protégés les "intérêts bien compris " des personnes dans quatre des systèmes évoqués précédemment (France, RFA, Suède, Victoria).

En France, donneurs et médecins sont très protégés. Le donneur ne court aucun risque, contrepartie logique du fait qu'il perd tout contrôle sur l'utilisation de ses paillettes, et n'est pas informé des résultats de ses dons. Les médecins tiennent une position confortable, grâce à une solide tradition de confidentialité : leurs obligations sont des obligations de moyens, ils sélectionnent les donneurs selon des règles internes à la profession, et ne sont pas menacés d'avoir à révéler l'identité des parties. La familleIAD est en position plus précaire. Le couple qui sollicite l'insémination doit se plier aux règles d'une déontologie médicale qui lui est imposée. La clause d'anonymat le préserve de toute irruption du donneur, mais lui interdit de pouvoir révéler jamais à l'enfant qui est son père génétique. Ce qui n'empêche pas le mari d'être investi d'une paternité fragile, puisque contestable au nom de l'absence de lien génétique, même s'il a consenti à l'IAD, ce qui rend son consentement dérisoire. La femme doit accepter que le géniteur de son enfant disparaisse derrière le personnage du médecin qui choisit la paillette de sperme. Elle doit en outre supporter de ne pas connaître cet homme dont le sperme, introduit dans son vagin, a permis la conception d'un enfant qu'elle va porter neuf mois en ignorant la moitié de sa provenance. Elle possède, enfin, un pouvoir ambivalent sur son mari, puisqu'elle peut contester sa paternité, elle dont la maternité est incontestable. L'enfant, quant à lui, se voit dénier le droit de savoir son origine exacte : ou bien il restera toute sa vie "protégé " par le mutisme de ses parents, ou bien il saura que sa conception est due au don anonyme d'une goutte de sperme qui a transité par une banque et par la congélation (qui abolit la dimension temporelle). Est-il si simple de faire le deuil de "rien"? Nous pensons qu'on ne peut faire le deuil que du connu (par exemple, d'un père mort, disparu, ou indigne). Nous avons indiqué ailleurs les risques d'irrepérage paternel [13].

Il faut noter, dans ce type de système, l'importance considérable du secret. Ce secret abrite un droit à l'intimité, forme de liberté négative, consistant à être préservé contre l'ingérence d'autrui dans ses affaires privées. La non-circulation des informations, dans le même temps, limite la liberté positive et les responsabilités. Chacun répond de ses actes devant sa conscience, la communication est minimale. Ainsi le donneur qui aurait sciemment dissimulé une tare ne peut être poursuivi, il est couvert par l'anonymat; mais il ne peut ni connaitre, ni assumer, les conséquences de son geste. Peu de guidance psychologique, pas de suivi : le médecin "ne doit pas s'immiscer dans la décision de procréation qui appartient au couple " [9], il ne doit pas chercher à savoir comment se développe l'enfant; la recherche sur les enfantsIAD est presque inexistante. L'intérêt de l'enfant, plus ou moins confondu avec celui de sa famille, n'implique ni le droit à la vérité, ni le droit de revendiquer un père (ou une mère); il implique le droit au secret protecteur. On craint que la révélation d'une double paternité biologique et sociale ne fasse " éclater la famille". Notons que la collectivité ne se hâte cependant pas de prévenir les ennuis qu'entraîne une levée accidentelle du secret, comme si elle tenait à ce que les libertés sus-mentionnées s'exercent, pour les familles bénéficiant d'un " enf ant de la médecine", dans une relative insécurité : en général, les juristes français ne souhaitent actuellement consolider aucune filiation " artificielle " par un statut juridique.

En RFA, les brèches ouvertes dans la confidentialité accentuent l'insécurité, en l'absence de renfort juridique. L'épouse du donneur n'a rien à dire, bien qu'elle ait d'éventuelles conséquences à supporter. Une menace aléatoire pèse sur le donneur, difficilement acceptable, puisqu'il ne dépend pas de lui que la mésentente du couple receveur déclenche une procédure judiciaire 
qui dévoile son identité, et transfère sur lui les droits et devoirs paternels. Certes le médecin doit l'avoir informé des conséquences possibles, quoique improbables, de son don de paternité. Mais le médecin luimême est dans une position délicate. Il faut bien qu'il trouve des donneurs s'il veut pratiquer l'IAD, et il y a une demande pour l'IAD. Etant mal couvert par le secret médical, il n'est pas à l'abri d'une condamnation à des dommages et intérêts en cas de procédure judiciaire. (En Suisse, il n'est pas exclu qu'il soit un jour mis en cause par l'enfant, par exemple pour l'atteinte aux droits de la personne que constitue le fait de naître sans père, en cas d'insémination d'une femme seule.) Les parents-IAD n'ont pas non plus une position très confortable. La filiation avec le père "apparent" peut être dénoncée par plusieurs personnes, y compris l'enfant, mais non par la mère. L'enfant est en principe le gagnant de cette histoire. C'est au nom de ses " droits fondamentaux " que l'anonymat du donneur est jugé "immoral ". Ces droits fondamentaux sont le droit à un père, et le droit à la vérité (la "vraie" filiation paternelle étant la filiation génétique). La fuite de la clientèle IAD vers la clandestinité des cabinets médicaux privés, depuis le verdict áu BGH en I983, a évidemment rendu très difficile tout contrôle, et toute enquête objective sur la situation de ces enfants.

Le coût psychologique, pour toutes les parties, du dévoilement brutal, par une enquête judiciaire de la vérité biologique, est considérable. Quelles valeurs très fortes défendon au prix de ce risque? En assimilant l'enfant IAD à un " enfant de l'adultère ", on tente, semble-t-il, de lui tisser des liens humains. L'idée que le donneur de gamètes puisse contribuer à faire un enfant " en dehors de toute communication humaine " et sans prendre aucune responsabilité, est mal supportée en RFA. "La franchise d'une naissance par mère de substitution effraie moins que le mensonge d'une naissance IAD avec donneur anonyme " [7]. $\mathrm{Au}$ lieu qu'en France, pour ne pas troubler l'intimité familiale, on détache de la personne du donneur son potentiel génétique, apporté au couple demandeur comme un don médical, on résiste en Allemagne fédérale à la réification d'une partie de la personne, et on accepte la souffrance qui peut résulter d'une re-sexualisation des techniques procréatives. Pas plus qu'en France on ne paraît souhaiter, pour le moment, atténuer cet inconf ort par des mesures juridiques.

Responsabilité et droit à la vérité : les exemples suédois et de l'état de Victoria.

Démembrement de la filiation, étalée à tous les regards; chosification technologique de l'être humain à ses origines; ce sont les périls de la "procréatique ". Le second est bien analysé par C. Labrusse-Riou [14]. La France et la RFA sont des exemples de pays qui refusent l'un ou l'autre. L,a Suède, l'état du Victoria, ont plutôt cherché à les encadrer par des législations spéciales, qui, en régulant les flux d'information, donnent aux individus plus de liberté positive (au prix d'une perte d'intimité), et plus de sécurité psychologique (au prix d'une directivité forte)

En Suède, le donneur actuel (postloi de 85 ) est à la fois protégé par la loi (la paternité de l'enfant-IAD ne peut lui échoir), et fortement responsabilisé en tant que donneur de gènes : en acceptant le risque d'une confrontation éventuelle avec l'enfant, lui et sa compagne font une démarche qui n'est pas neutre, ni pour eux-mêmes, ni pour leurs propres enfants. A la lumière de ce qu'on sait sur la psychodynamique du don de sperme [15], nous pensons qu'en contrepartie il peut tirer des bénéfices psychologiques de cette position, et que (passé l'effroi qu'elle a pu susciter) la loi suédoise n'est pas injuste pour les donneurs. Les médecins ont une charge lourde : outre l'intervention proprement médicale, ils doivent apprécier la situation psycho-sociale du couple, un peu comme les services sociaux le font dans les enquêtes préliminaires à l'adoption. De plus, il leur incombe de recueillir les consentements avec soin, celui du mari étant légalement irréversible une fois les inséminations commencées. La loi suédoise a été explicite- ment votée dans l'intérêt de l'enfant: celui-ci ne risque de se retrouver ni sans père, ni nanti d'un autre père que le premier, et il a le droit de savoir comment il a été conçu. Les textes d'application prévoient en outre quelles précautions doivent être prises pour que, adolescent, s'il le désire, il puisse accéder à la révélation de l'identité du donneur sans traumatisme inutile (l'hôpital ne doit pas communiquer de données sans que le demandeur ait eu un entretien avec un professionnel compétent des services sociaux). Il peut sembler injuste que seul l'enfant ait un droit d'accès au dossier (et non pas sa mère, par exemple). Les parents dans le système suédois sont fortement encadrés, incités à vivre l'IAD comme un acte avouable, normal, révélable à l'enfant. On peut se demander si la pédagogie médicale suffit à aplanir la difficulté d'assumer entre soi et devant l'enfant cette " mise à plat " de la stérilité du père, et de l'immixtion d'un tiers dans l'intimité de la procréation. Cette réserve émise, il nous semble que le système suédois, malgré une certaine raideur, assure aux protagonistes une égalité de droits.

Concevoir la parenté-IAD sur le modèle de l'adoption plénière, malgré la différence des situations (parents en manque d'enfant, contre enf ant en manque de parents), c'est lui conférer une acceptabilité sociale : la filiation adoptive est noble. Cette respectabilité se paie par un contrôle strict (procédure réservée aux hôpitaux publics, accordée seulement aux couples stables, dans des indications médicales; pas de banques de sperme à but lucratif, pas d'insémination post mortem). Elle implique aussi pour les individus une véritable socialisation, ou sublimation, de la sexualité. Mais honorabilité n'est pas conformisme. La loi prévoit résolument que l'enfant a le droit d'être informé, dans son intérêt. L'idée qu'il n'est pas éthique de maintenir des habitudes dont la recherche montre qu'elles sont nocives pour le développement de la personne suggère qu'on valorise la connaissance scientifique. La possibilité de poursuivre une recherche sur le devenir de "l'adoption-IAD" est 
cependant limitée par le fait que la loi suédoise restreint au seul enfant l'accès au dossier.

L'état de Victoria se singularise par l'importance qu'il attache au consentement des donneurs de gamètes, et de leurs conjoint(e)s. Les donneurs peuvent fixer certaines conditions d'utilisation de leurs paillettes, les modifier en cours de route. Il est clairement entendu qu'ils donnent plus qu'un simple capital génétique, qu'ils peuvent avoir un engagement émotionnel qui les conduit à évoluer dans la compréhension de la finalité de leur geste, ou à avoir envie de savoir ce qu'il advient de leur don - ils sont des hommes, non des étalons. En face d'eux, la famille receveuse est traitée avec les mêmes égards, quant à son besoin de garder la mémoire d'un lien au donneur. La loi du Victoria prévoit en outre que tous les protagonistes reçoivent une guidance psychologique particulière. Les médecins, garants du sas par lequel transitent les informations non identifiantes entre donneurs et receveurs, sont très encadrés. Outre les comités médicaux et instances hospitalières, ils sont couverts par une autorité administrative (Ministre, ou Secrétaire de la Health Commission), qui tranche en cas de conflit entre les parties. Il existe, pour les litiges, un Standing Reviem and Advisory Committee [3]. Ce dernier est une commission composée de huit membres : un philosophe, deux médecins, deux représentants des familles spirituelles, un travailleur social, un juriste, un représentant de la fonction publique. Notons l'analogie avec la composition de la commission Warnock, analogie que l'on retrouve d'ailleurs dans les mesures préconisées. Mais, à la différence des comités européens qui n'ont qu'une voix consultative, il s'agit ici d'une instance de décision, qui gère un système manifestement inspiré par le double souci de traiter tous les intervenants comme des personnes humaines, ni trop protégées, ni trop exposées, et de ne pas laisser la procréation artificielle se développer sans contrôle.

Responsabilité médicale et prise en charge collective.

A la différence de la Suède, qui s'en remet aux médecins hospitaliers pour assurer la bonne marche de l'IAD dans les limites d'acceptabilité fixées par la loi, l'état du Victoria donne l'exemple d'une véritable prise en charge collective de la gestion des techniques de fécondation artificielle, comme si cette communauté australienne avait pris conscience que les enjeux actuels de la procréatique sont trop graves pour être laissés aux choix des individus, fussent-ils médecins. (Banques de sperme, d'ovules, d'embryons : les médecins auront bientôt le pouvoir de fabriquer des " enf ants de l'espèce " en dehors de tout lien personnel de filiation.) "Les médecins ne sont pas dispensés de suivre les standards éthiques de la communauté. Leur déontologie n'est pas une morale séparée, mais un aspect de notre morale. Décider de ce qu'ils doivent faire ou ne pas faire relève des principes éthiques de l'ensemble de la société " [2]. Ces principes exigent que l'information circule, que les risques psychologiques soient minimisés pour tous les intervenants, et que les liens humains soient préservés autant qu'ils peuvent l'être. Les informations (non identifiantes) contenues dans les registres tenus par les hôpitaux sont accessibles, non seulement aux parties concernées, mais à d'autres personnes " autorisées", possiblement des chercheurs. Des peines sont prévues pour ceux qui s'égareraient sur des voies prohibées : clonage, hybridation, recherche sur l'embryon, mais aussi fécondations successives d'une femme avec du sperme (ou des embryons) de donneurs différents (pratique habituelle en France pour l'IAD). Où l'on voit que, loin d'être réduit à une goutte de semence, le donneur devient ici un partenaire de la famille, avec, sinon une identité, du moins une silhouette individuelle.

Système pilote pour petit pays seulement? Expérience intéressante à suivre, en tout cas. "Cessons de nous auto-protéger, place à l'intérêt de l'enfant ", écrivait G. Annas dès I979 [12]. Nous dirions aujourd'hui qu'à travers le souci de l'enfant se fait jour le souci de ne pas laisser l'activité procréatrice de l'espèce se déshumaniser

\section{Summary}

Two international colloquiums on "artificial procreation, genetics and the law" were held in I 985, in Lausanne and Paris respectively. For these occasions a comprehensive body of laws and regulations relating to human artificial procreation was collected, which evidenced a variety of possible solutions to the problems faced today by sterile parents, gametes donors, "artificial" children, the medical profession, and society in general. The authors comment on the positions offered to parties in different cultural settings. In particular, they review the cases of France and Federal Republic of Germany, Sweden, the state of Ontario (Canada), and the state of Victoria (Australia).

\section{TIRES A PART}

G. Delaisi de Parseval : 118 , rue de Vaugirard, 75006 Paris. 